\title{
BASIDIOMICETES ENDOFÍTICOS DE MADERA EN PLATANUS ACERIFOLIA (PLATANACEAE) DE ARGENTINA: NOTAS Y ESTUDIOS DE CULTIVO
}

\author{
CAROLINA A. ROBLES ${ }^{1}$, SILVIA E. LOPEZ y CECILIA C. CARMARÁN
}

\begin{abstract}
Summary: Basidiomycetous wood endophytes from Platanus acerifolia (Platanaceae) of Argentina: notes and culture studies. The knowledge of local fungal strains' features increases our ability to make use of their properties and to implement, in consequence, new and better ways of using the native biological resources. To contribute to the knowledge of these organisms, in this work we present descriptions and cultural studies of 5 species previously reported as endophytic basidiomycetes: Coprinopsis cinerea, Granulobasidium vellereum, Inonotus rickii, Phanerochaete chrysosporium and Trichosporon sporotrichoides, isolated from wood of London plane trees (Platanus acerifolia) in Buenos Aires City, Argentina. Coprinopsis cinerea is described in culture for the first time and differences in cultures of $G$. vellereum and $I$. rickii with previous reports are listed. Culture characters of $P$. chrysosporium and $T$. sporotrichoides did not show appreciable differences with previous descriptions.
\end{abstract}

Key words: Urban trees, basidiomycetes, wood endophytes.

\begin{abstract}
Resumen: Conocer las características de cepas fúngicas locales aumenta nuestra capacidad de poder hacer uso de sus propiedades e implementar, en consecuencia, nuevas y mejores formas de aprovechamiento de los recursos biológicos nativos. Con el fin de contribuir al conocimiento de estos organismos, en este trabajo se presentan descripciones detalladas y estudios de cultivo de 5 especies previamente reportadas como basidiomicetes endofíticos: Coprinopsis cinerea, Granulobasidium vellereum, Inonotus rickii, Phanerochaete chrysosporium y Trichosporon sporotrichoides, aisladas como endofitos de madera de plátanos de sombra (Platanus acerifolia) en la Ciudad de Buenos Aires, Argentina. Coprinopsis cinerea es descripta por primera vez en cultivo y se señalan las diferencias que presentan los cultivos de G. vellereum e I. rickii con reportes previos. Los caracteres de cultivo de $P$. chrysosporium y $T$. sporotrichoides no presentaron diferencias apreciables con descripciones previas
\end{abstract}

Palabras clave: Árboles urbanos, basidiomicetes, endofitos de madera.

\section{INTRODUCCIÓN}

Los endofitos se definen como organismos, hongos y bacterias, que colonizan tejidos vegetales, persistiendo en parte o todo el ciclo de vida de la planta sin causar síntomas de enfermedad (Schulz \& Boyle, 2006; Arnold, 2007). Se ha sugerido que ciertos endofitos podrían ser patógenos en estado

1 PROPLAME-PRHIDEB-CONICET, Departamento de Biodiversidad y Biología Experimental, Facultad de Ciencias Exactas y Naturales, Universidad de Buenos Aires, Ciudad Universitaria, PB II, 4to piso, 1428EHA, Ciudad Autónoma de Buenos Aires, Argentina; carorobles@bg.fcen.uba.ar (autor corresponsal). de latencia, por ejemplo hongos degradadores de madera que se encuentran en una etapa de su ciclo en la cual no utilizan la madera como fuente nutricional (Oses et al., 2008; Parfitt et al., 2010).

Si bien la mayoría de los endofitos conocidos pertenecen al Phylum Ascomycota (Angelini et al., 2012), existe un número creciente de trabajos en los cuales se reconocen especies de basidiomicetes como endofitos en madera (Baum et al., 2003; Oses et al., 2008; Pinruan et al., 2010; Martin et al., 2015). En Argentina solo se conocen dos trabajos donde se reportan basidiomicetes aislados como endofitos de madera. De Errasti et al. (2010) aislaron Coprinellus micaceus (Bull.) Vilgalys, Hopple \& Jacq. Johnson como endofito de Broussonetia papyrifera (L.) 
L'Hér. ex Vent. y Celtis occidentalis L. Más recientemente, Robles et al. (2015) reportaron 41 aislamientos pertenecientes a 5 especies de basidiomicetes recuperados como cepas endofíticas en árboles urbanos de Platanus acerifolia Aiton (Willd.) (plátano de sombra). Teniendo en cuenta la poca disponibilidad de información en el ámbito nacional sobre la biodiversidad de endofitos de maderas y, al mismo tiempo, de basidiomicetes endofíticos, se consideró de interés estudiar en detalle las cepas obtenidas, brindando descripciones detalladas de los cultivos puros y observaciones sobre las especies reportadas.

Este trabajo tiene como objetivo contribuir al conocimiento de la diversidad de especies endofíticas de madera en Argentina.

\section{Materiales y Métodos}

Las cepas estudiadas se hallan depositadas en la Colección de Cultivos de la Facultad de Ciencias Exactas y Naturales de la Universidad de Buenos Aires (BAFCcult). Las mismas provienen de muestreos realizados durante los años 2010 y 2011 en la Ciudad Autónoma de Buenos Aires, en 4 sitios con diferente nivel de disturbio antrópico: sitio A

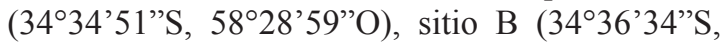
$\left.58^{\circ} 23^{\prime} 54^{\prime \prime O}\right)$, sitio C (34³9 25”S, 58²9'15”O) y sitio D (34³2'41'’S, 58²6’39”O) (Fig. 1). Las diferencias en el nivel de disturbio de estos sitios son detalladas en Robles et al. (2015).

En los sitios considerados se tomaron muestras de madera de árboles asintomáticos. En cada uno de ellos, se removió una sección de corteza (ca. $3 \mathrm{~cm} \times 3 \mathrm{~cm}$ ) y luego se colectaron muestras de madera cilíndricas, de aproximadamente $10 \mathrm{~cm}$ de largo y visualmente sanas, por medio de un taladro de incremento (Suunto, Vantaa, Finlandia). El taladro fue esterilizado superficialmente con etanol $70 \%$ luego de cada uso. Cada orificio producido en los árboles fue sellado con sellador de silicona para minimizar la infección posterior al muestreo. Las muestras fueron rociadas con etanol 70\% y guardadas en tubos Falcon de $15 \mathrm{ml}$ para su posterior procesamiento. En el laboratorio las muestras fueron cortadas en fragmentos de ca. $0,5 \mathrm{~cm}$ de largo, y cada uno fue esterilizado superficialmente $(50 \%$ etanol por 30 segundos, $2 \%$ masa/volumen $\mathrm{NaOCl}$ por 1 minuto, y $50 \%$ etanol por 30 segundos), flameados y colocados en placas de Petri de $9 \mathrm{~mm}$ de diám con extracto de malta $2 \%$ con cloranfenicol $(100 \mathrm{mg} / \mathrm{l})$ o un medio selectivo para basidiomicetes (BDS). Los fragmentos fueron incubados en oscuridad a $24{ }^{\circ} \mathrm{C}$ y examinados cada 2-3 días para detectar crecimiento fúngico (Robles et al., 2015).

Los aislamientos fueron caracterizados a partir de claves de caracteres de cultivo para Basidiomycota y bibliografía específica (Nobles, 1965; Stalpers, 1978; Deschamps \& Wright, 1997). Para esto se siguió la metodología de Nobles (1965) a partir de caracteres macro y micromorfológicos. La numeración de las claves patrón presentadas en las descripciones de las especies, corresponden a la adoptada por Deschamps \& Wright (1975). Las estructuras micromorfológicas fueron medidas con un aumento de $400 \mathrm{X}$ y $1000 \mathrm{X}$ con un microscopio Zeiss Axioskop (Oberkochen, Alemania) equipado con una cámara digital Olympus modelo C-5060 Wide Zoom 5.1 megapíxeles (Tokio, Japón). Se utilizaron los medios de montaje $\mathrm{KOH} 5 \% \mathrm{p} / \mathrm{v}$ y floxina $1 \% \mathrm{p} / \mathrm{v}$ para microscopia óptica. Para la nomenclatura de las especies se siguió a la base de datos MycoBank (http://www.mycobank. org/). A continuación de los números BAFCcult correspondientes a los cultivos estudiados se brindan los números de GenBank asociados a las secuencias obtenidas, reportadas en Robles et al. (2015). Las secuencias obtenidas permitieron corroborar las identificaciones morfológicas.

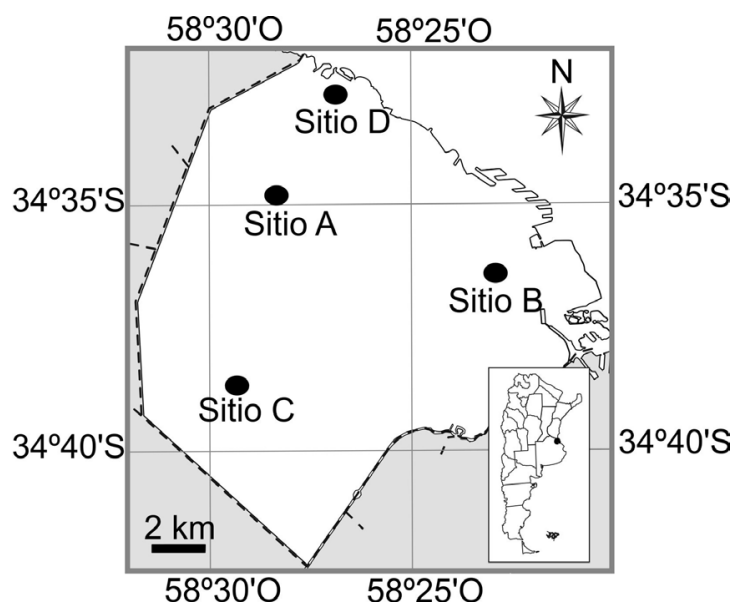

Fig. 1. Sitios de muestreo en la Ciudad Autónoma de Buenos Aires. 

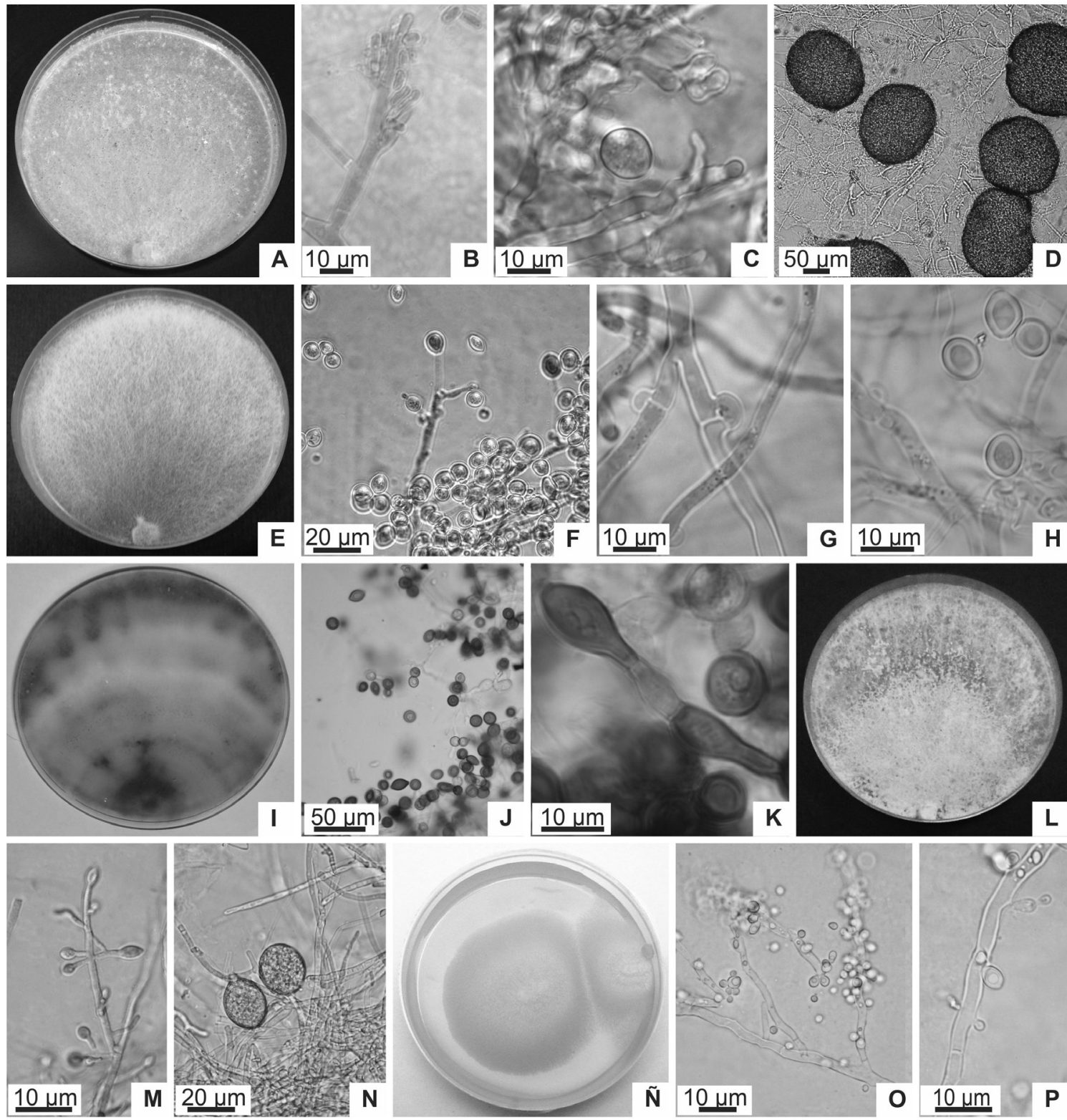

Fig. 2. Paneles $A-D$ : Coprinopsis cinerea. $A$, aspecto de la colonia al término de la sexta semana en medio de Nobles. B, formación de artrosporas. C, presencia de clamidosporas. D, esclerocios. Paneles E-H: Granulobasidium vellereum. E, aspecto de la colonia al término de la sexta semana en medio de Nobles. F, vista general de hifas generativas y clamidosporas. $\mathrm{G}$, hifas generativas fibuladas. $\mathrm{H}$, clamidosporas, con pared celular engrosada. Paneles I-K: Inonotus rickii. I, aspecto de la colonia al término de la sexta semana en medio de Nobles. J, vista general de hifas generativas y clamidosporas. K, formación de clamidosporas en cadena. Paneles L-N: Phanerochaete chrysosporium. L, aspecto de la colonia al término de la sexta semana en medio de Nobles. M, formación de conidios. N, clamidosporas y micelio vegetativo. Paneles $\tilde{\mathrm{N}}$-P: Trichosporon sporotrichoides. $\tilde{\mathrm{N}}$, aspecto de la colonia luego de 2 semanas en Extracto de Malta $2 \%$. $\mathrm{O}$, hifas con formación de conidios. $\mathrm{P}$, detalle de formación de conidios. 


\section{Resultados}

\section{Agaricales \\ Psathyrellaceae \\ Coprinopsis cinerea (Schaeff.) Redhead,} Vilgalys \& Moncalvo; Taxon 50(1): 227; 2001. (Fig. 2A-D).

Descripción del cultivo puro. Caracteres macroscópicos: Crecimiento lento, cubriendo la caja a la quinta semana. Micelio blanco, hialino, con textura algodonosa laxa. A partir de la segunda semana comienzan a observarse pequeños esclerocios cercanos a la zona del inóculo, frecuentemente rodeados por gotas, que les dan un aspecto brillante. En la cuarta semana, los esclerocios son más abundantes y se ubican concéntricamente en el centro de la colonia. Borde regular. Reverso inalterado. Sin olor característico.

Caracteres microscópicos: Micelio aéreo con hifas hialinas, con septos simples en su gran mayoría, muy escasas fíbulas, teñibles con floxina, 2-4 $(4,5)$ $\mu \mathrm{m}$ de diám. Desde la primera semana se observan abundantes artrosporas, 4-6 (7,5) x 1,5-2 $\mu \mathrm{m}$ a partir de células de 13-25 x 2-4 $\mu$ m. Simultáneamente, en algunos cultivos aparecen células ensanchadas, cada vez más abundantes a partir de la segunda semana, 140-170 (200) x 130-150 (180) $\mu \mathrm{m}$, las cuales se agrupan para formar esclerocios. Esclerocios con las células de la pared de color castaño oscuro: 8-15 x 5-8 (10) $\mu \mathrm{m}$, las de la médula son hialinas y de mayor tamaño: 19-41 x 4-10 $\mu \mathrm{m}$. En ambos casos presentan formas irregulares y están dispuestas en forma de rompecabezas. En la cuarta semana se observan clamidosporas, escasas, cerca de la zona del esclerocio 10-22 x 6-12 $\mu \mathrm{m}$. En el micelio sumergido se observan hifas más delgadas y retorcidas 1,5-3 $(3,5) \mu \mathrm{m}$; si bien se observan artrosporas sueltas, no fue posible detectar su formación.

\section{Clave patrón. 1 (2). 6. 23. 35. 36. 38. 45. 53.}

Observaciones: Se obtuvieron 2 aislamientos de esta especie a partir de 1 árbol del sitio A (Fig. 1), el cual constituye un área residencial, con baja presencia de comercios e industrias y tránsito vehicular. Las características registradas en el estudio de cultivos coinciden con el anamorfo de Coprinopsis cinerea (v. gr., Hormographiella aspergillata Guarro, Gené \& De Vroey). Si bien no se han encontrado trabajos sobre estudios de cultivo en base a la metodología de Nobles para esta especie, Guarro et al. (1992) y Kües (2000) presentaron una descripción de los caracteres micromorfológicos en cultivo de $C$. cinerea, bajo el nombre de $H$. aspergillata. Coprinopsis cinerea es un basidiomicete heterotálico descripto como cosmopolita y asociado generalmente a compost y aguas residuales (Seifert et al., 2011), estiércol y chips de madera (Keirle et al., 2004). Se distinguen dos tipos de micelio en su ciclo de vida, el monocarionte afibulado y el dicarionte fibulado, presentando ambos un amplio potencial de crecimiento. Puede formar clamidosporas, oídios, esclerocios, cordones miceliales y pseudorrizas (Kües, 2000). Hasta el momento se lo ha reportado como endofito solo en P. acerifolia de Argentina (Robles et al., 2015). Ese registro constituye la primera cita de esta especie para la Argentina.

Material examinado: ARGENTINA; Ciudad Autónoma de Buenos Aires; Parque Chas; 34³5'10'S; 58²8'56”O; 4-VI-2010; Robles C. s.n.; BAFCcult 4361; GenBank KC881188.

\section{Agaricales \\ Cyphellaceae}

Granulobasidium vellereum (Ellis \& Cragin) Jülich; Persoonia; 10 (3): 328; 1979. (Fig. 2E-H).

Descripción del cultivo puro. Caracteres macroscópicos: Presenta un crecimiento rápido, cubriendo la caja al término de la segunda semana. La mata miceliana inicialmente es hialina, tornándose levemente crémea a partir de la cuarta semana, en especial cerca del inóculo. Micelio en un principio algodonoso, sobre todo hacia el centro de la colonia, algo apretado al sustrato en el borde de la colonia. A partir de la segunda semana, el número de clamidosporas aumenta hasta prevalecer sobre el micelio dando a la colonia un aspecto pulverulento y laxo. En algunos cultivos se forman pequeños cúmulos de clamidosporas que sobresalen del resto de la colonia. Margen regular. Reverso inalterado a lo largo del estudio. No se detectó olor característico.

Caracteres microscópicos: Hifas fibuladas, hialinas, con pared delgada, teñibles con floxina, 2-4 $\mu \mathrm{m}$ de diám.; clamidosporas abundantes, terminales 
e intercalares, con forma de limón y/o globosas. Estas aparecen a partir de la primera semana en toda la caja, 6-11 x 6-9 $\mu \mathrm{m}$, con paredes de 1-2 $\mu \mathrm{m}$ de espesor. El micelio sumergido presenta los mismos elementos que el micelio aéreo, hifas generativas: 2-4 $\mu \mathrm{m}$ de diám., clamidosporas: 6-10 x 5-8 $\mu \mathrm{m}$.

Clave patrón. 1. 3. 7. 34. 36. 38. 42. 51. 54. 60.

Observaciones: Se obtuvieron 11 aislamientos de esta especie, a partir de un total de 5 árboles del sitio A (Fig. 1). La descripción coincide en su mayor parte con las realizadas por Nobles \& Nordin (1955) y Stalpers (1978). Difiere en que en estas descripciones se indica que frecuentemente aparecen basidiomas en el cultivo antes de la sexta semana, aunque esto no se observó en ninguno de los cultivos estudiados en este trabajo. Granulobasidium vellereum es una especie común en Europa, particularmente en Italia. Diversos autores la han citado como asociada a una pudrición blanca sobre tocones y árboles vivos de angiospermas (Nobles \& Nordin, 1955; Stalpers, 1978; Nakasone, 1990) y es común sobre Ulmus sp. (Bernicchia et al., 2007). En Sudamérica ha sido citada para Brasil (Ramos Bononi et al., 2008) pero esta especie no había sido citada previamente para la Argentina. Hasta el momento se lo ha reportado como endofito solo en $P$. acerifolia de Argentina (Robles et al., 2015).

Material examinado: ARGENTINA; Ciudad Autónoma de Buenos Aires; Villa Urquiza; 34³4'54”S; 58²9'14”O; 16-VII-2010; Robles C. s.n.; BAFCcult 4362; GenBank KC881192; ídem; 34³4'48'S; 58²8'54'O; 16-VII-2010; BAFCcult 4363; GenBank KC881194; ídem; 10-VIII-2010 BAFCcult 4364; ídem, 34 34' 36”S; 58²9'44”O; 10-VIII-2010; BAFCcult 4365; GenBank KC881191; ídem; 34³4'48”'S; 58²9'14”O; 10-VIII-2010; BAFCcult 4366; GenBank KC881190; ídem; 34³5'10’'S; 58²8'56”'O; 10-VIII-2010; BAFCcult 4367; GenBank KC881193.

\section{Hymenochaetales \\ Hymenochaetaceae}

Inonotus rickii (Pat.) D.A. Reid; Kew Bull; 12(1): 141; 1957. (Fig. 2I-K).

Clave patrón. 2.6.8.18.34.37.39.46.(47).53.54. Para la descripción del cultivo ver Wright \& Iaconis (1955).
Observaciones: Se obtuvieron 5 aislamientos a partir de un total de 3 árboles del sitio A (Fig. 1). El estudio de los cultivos coincide con la descripción de Wright \& Iaconis (1955), excepto por su velocidad de crecimiento, ya que estos autores mencionan que los cultivos cubrían la caja al término de la segunda semana, y en este estudio lo hicieron a la sexta semana o no llegaron a cubrir las cajas al término del mismo. Sin embargo se han descripto cepas que presentan una velocidad de crecimiento menor, cubriendo la caja al término de las 6 semanas de estudio (Robles et al. 2011). Inonotus rickii es un hongo de pudrición blanca ampliamente distribuido en zonas tropicales y subtropicales. En zonas templadas, sin embargo, se encuentra asociado principalmente a ambientes urbanos. Además de haber sido encontrado afectando árboles urbanos de Sudamérica (Gottlieb et al., 2002; Robles et al., 2011), se lo ha reportado en varios países de Europa (Melo et al., 2002; Annesi et al., 2003; Intini \& Tello, 2003) y en Norteamérica (Farr et al., 1989). En Argentina ha sido previamente citado sobre P. acerifolia (Robles et al., 2011), Acacia melanoxylon R. Br., Acer negundo L., Casuarina cunninghamiana Miq., Celtis australis L. y Lippia citriodora Kunth (Gottlieb et al., 2002), evidenciando su amplio rango de hospedantes. Existen algunos trabajos en los cuales se han aislado cepas endofíticas de Inonotus colonizando madera de Podocarpus saligna D. Don en Chile (Oses et al., 2008) y tallos de Theobroma cacao L. en Costa Rica (Crozier et al., 2006), sugiriendo que diversas especies de este género podrían tener la capacidad de colonizar asintomáticamente la madera en árboles vivos.

Material examinado: ARGENTINA; Ciudad Autónoma de Buenos Aires; Villa Urquiza; 34³4'48”'S; 58²9’14”O; 10-VIII-2010; Robles C. s.n.; BAFCcult 4301; ídem; 3434'49"S; 58 29'14"O; 1-IX-2010; BAFCcult 4302; ídem; 34³4'54”'S; 58²9'14'O; 10-VIII-2010; BAFCcult 4303.

\section{Polyporales \\ Phanerochaetaceae}

Phanerochaete chrysosporium Burds.; Mycotaxon; 1(2): 124; 1974. (Fig. 2L-N).

Descripción del cultivo puro. Caracteres macroscópicos: Crecimiento rápido, cubriendo 
la caja al término de la primera semana. Mata miceliana blanca, al principio esponjosa, luego adoptando un aspecto pulverulento debido a la gran esporulación. Margen regular. Reverso inalterado a lo largo del estudio. No se detectó olor característico.

Caracteres microscópicos: Hifas hialinas estériles, 2,5-7 (8) $\mu \mathrm{m}$ de diám, con septos simples y de pared delgada, hifas fértiles más delgadas, $1 \mu \mathrm{m}$ de diám, con septos simples. Presencia de algunas hifas de 4-7 $\mu \mathrm{m}$ de diám, con pared gruesa: 1,5-2,5 $\mu \mathrm{m}$ de espesor. Conidios laterales y terminales, solitarios, unicelulares, hialinos, lisos o con pequeñas equinulaciones, muy abundantes a la tercera semana, al principio con pared delgada y luego desarrollando una pared más gruesa, obovoides a piriformes, 8-10 x (5) 6-7 $\mu \mathrm{m}$, originados a partir de un desarrollo simpodial de los conidióforos. Hacia la cuarta-quinta semana el micelio aéreo se compone principalmente de conidios, con escasa presencia de hifas. Clamidosporas escasas, se observan mayormente a partir de la sexta semana, 13-39 $\mu \mathrm{m}$ de diám. Hacia la quinta semana se observan hifas con partes del citoplasma colapsado, como si fueran a formar artrosporas.

Clave patrón. 1. 6. (7). 33. 34. 35. 36. 40. 41. (51). 53. 54. 55. (57). 59.

Observaciones: Se obtuvo solamente un aislamiento de esta especie, en el sitio C, el cual comprende un área residencial con una importante presencia de industrias y comercios (Fig. 1). La descripción coincide con la realizada por Stalpers (1978) y Nakasone (1990). Esta especie causa una pudrición blanca de tronco y ramas de angiospermas y gimnospermas (Burdsall \& Elysn, 1974) y en Argentina ha sido utilizada en trabajos de evaluación enzimática (Levin et al., 2004), aunque no existen registros previos de cepas aisladas en el país. Ha sido reportada para Norteamérica y Europa (Bundsall \& Elysn, 1974; Nakasone, 1990). El género Phanerochaete ha sido citado como endofito de tallo de T. cacao (Crozier et al., 2006) y como endofito de madera y hoja de Hevea Aubl. (Martin et al., 2015), pero no se habían encontrado reportes de Phanerochaete chrysosporium como endofito de madera (cfr. Robles et al., 2015). Esta especie no había sido registrada anteriormente para $P$. acerifolia, aunque sí había sido reportada colonizando Platanus wrightii S. Watson como degradador (Burdsall \& Eslyn, 1974).

Material examinado: ARGENTINA; Ciudad Autónoma de Buenos Aires; Mataderos; 3439'22”S; 58²9'16”O; 11-XI-2011; Robles C. s.n.; BAFCcult 4415; GenBank KC881189.

\section{Tremellales \\ Trichosporonaceae}

Trichosporon sporotrichoides (Oorschot) Oorschot\& de Hoog; Antonie van Leeuwenhoek; 47(4): 364; 1981. (Fig. 2N-P).

Descripción del cultivo puro: Crecimiento lento, mata miceliana de color blanca o crémea, micelio sumergido o apretado contra el agar, de aspecto húmedo. Hifas sumergidas, con frecuencia desarticulándose por los septos, fértiles, fragmentos resultantes de la desarticulación: (1) 2-4 (6) x (8) 1123 (27) $\mu \mathrm{m}$. Conidios abundantes, solitarios, 1-5 por célula conidiógena, subhialinos, lisos y con paredes delgadas, la mayoría globosos, algunos ligeramente piriformes, 3-5 (7) x (1,5) 2-3 (4) $\mu \mathrm{m}$.

Observaciones: Se obtuvieron 22 aislamientos de esta especie: 1 aislamiento en el sitio A, 6 aislamientos en el sitio $\mathrm{C}$ (a partir de 4 árboles) y los 15 aislamientos restantes en el sitio D (a partir de 4 árboles). El sitio D se encuentra comprendido entre un área residencial y el parque natural de Ciudad Universitaria, y presenta escasos indicadores de disturbio antrópico (Fig. 1). La descripción coincide con la realizada por Van Oorschot (1980). El género Trichosporon Behrend comprende hongos mitospóricos, anamorfos de Tremellales (Seifert et al., 2011). Este género está ampliamente distribuido en la naturaleza y se los encuentra predominantemente en sustratos como suelo y madera en descomposición (Chagas-Neto et al., 2008). El género ha sido citado para la Argentina (Pajot et al., 2008). Existen pocos registros del mismo como endofito, en tejidos de almacenamiento y raíces (Götz et al., 2006; Isaeva et al., 2010), aunque no se lo registró como endofito de madera. Los aislamientos endofíticos obtenidos constituyen la primera cita para Argentina y sobre $P$. acerifolia.

Material examinado: ARGENTINA; Ciudad Autónoma de Buenos Aires; Núñez; 34³2’41"S; 58²6’39”O; 10-V-2011; Robles C. s.n.; BAFCcult 
4328; ídem; 10-V-2011; BAFCcult 4329; ídem; 10-V-2011; BAFCcult 4331; ídem; 34³9'27’'S; 58²9'14”O; 11-XI-2011; BAFCcult 4404; ídem; 34³9'22”'S; 58²9'16”'O; 18-IX-2011; BAFCcult 4405; ídem; 34³9'29”S; 58²9'7’O; 18-IX-2011; BAFCcult 4407; ídem; 34³9'27’S; 58²9'14”O; 11-XI-2011; BAFCcult 4408.

\section{Conclusiones}

Durante el desarrollo de un trabajo sobre potenciales relaciones entre endofitos de madera y hongos de pudrición en el arbolado urbano (Robles et al., 2015), se obtuvieron 41 cepas de basidiomicetes endofíticos. Sin embargo, en ese trabajo previo no se brindó información adicional de las 5 especies registradas, algunas de ellas primeros registros para Argentina, como son los casos de Coprinopsis cinerea, Granulobasidium vellereum y Phanerochaete chrysosporium. Teniendo en cuenta la casi inexistente información acerca de basidiomicetes endofíticos en nuestro país, se consideró de interés reportar el resultado de los estudios de cultivo in vitro de estas cepas. De las 41 cepas estudiadas, aquellas asignadas a las especies Granulobasidium vellereum e Inonotus rickii presentaron diferencias respecto a reportes previos, mientras que se describe por primera vez en cultivo a Coprinopsis cinerea.

La importancia de utilizar cepas locales de organismos, las cuales pueden presentan características morfológicas y fisiológicas particulares, ya ha sido considerada en diferentes estudios (Schwartz et al., 2006; Marulanda et al., 2009). El conocimiento integral de las características de cepas de poblaciones locales constituye una útil herramienta para estudios aplicados.

Este trabajo brinda información detallada de cepas obtenidas, a partir del muestro de madera de árboles urbanos, describiéndose 5 géneros de basidiomicetes, correspondientes a 5 familias y 4 órdenes diferentes. Martin et al. (2015) obtuvieron 90 unidades formadoras de colonias de basidiomicetes endofíticos a partir de madera de Hevea, los cuales correspondían a 9 órdenes, incluyendo a los 4 órdenes también representados en el presente trabajo (Agaricales, Hymenochaetales, Polyporales y Tremellales). Estos resultados indicarían que estos órdenes de basidiomicetes podrían tener una importante presencia en las comunidades endofíticas de madera.

Las especies Inonotus rickii y P. chrysosporium fueron registradas como endofitos de madera en árboles urbanos del país y son especies reconocidas como causantes de pudrición blanca en numerosos hospedantes. Diferentes trabajos (Promputtha et al., 2007; Parfitt et al., 2010; Martin et al., 2015) han sugerido la posibilidad de que algunas especies endofíticas puedan estar relacionadas con desarrollo de estadios tempranos de pudrición de la madera. La identificación de cepas endofíticas nativas de especies que tengan capacidad de actuar como degradadores de madera constituye una valiosa información para el conocimiento de las comunidades locales de hongos.

\section{Agradecimientos}

Este estudio fue financiado por el Consejo Nacional de Investigaciones Científicas y Técnicas (CONICET) (proyecto PIP 0846/ PIP1086) y la Universidad de Buenos Aires, Argentina (proyecto UBACYT x010). Publicación numero 211 PROPLAME-PRHIDEB-CONICET.

\section{Bibliografía}

ANGELINI, P. A. RUBINI, D. GIGANTE, L. REALE, R. PAGIOTTI \& R. VENANZONI. 2012. The endophytic fungal communities associated with the leaves and roots of the common reed (Phragmites australis) in Lake Trasimeno (Perugia, Italy) in declining and healthy stands. Fungal Ecol. 5: 683-693.doi http://dx.doi.org/10.1016/j. funeco.2012.03.001

ANNESI, T., R. COPPOLA \& E. MOTTA. 2003. Decay and canker caused by Inonotus rickii spreading on more urban tree species. Forest Pathol. 33: 405-412.

ARNOLD, A. E. 2007. Understanding the diversity of foliar endophytic fungi: progress, challenges, and frontiers. Fungal Biol. Rev. 21: 51-66.

BAUM, S., T. N. SIEBER, F. W. M. R. SCHWARZE \& S. FINK. 2003. Latent infections of Fomes fomentarius in the xylem of European beech (Fagus sylvatica). Mycol. Prog. 2: 141-148.

BERNICCHIA, A., G. SABINO \& S. PÉREZ GORJÓN. 2007. Aphyllophoraceous wood-inhabiting fungi on Abies alba in Italy. Mycotaxon 100: 185-188.

BURDSALL JR, H. H. \& W. E. ESLYN. 1974. A new 
Phanerochaete with a Chrysosporium imperfect state. Mycotaxon 1: 123-133.

CHAGAS-NETO, T. C., G. M. CHAVES \& A. L. COLOMBO. 2008. Update on the Genus Trichosporon. Mycopathologia 166: 121-132.

CROZIER, J., S.E. THOMAS, M. C. AIME, H. C. EVANS \& K. A. HOLMES. 2006. Molecular characterization of fungal endophytic morphospecies isolated from stems and pods of Theobroma cacao. Plant Pathol. 55: 783-91.

DE ERRASTI, A., C. C. CARMARÁN \& M. V. NOVAS. 2010. Diversity and significance of fungal endophytes from living stems of naturalized trees from Argentina. Fungal Divers. 41: 29-40.

DESCHAMPS, J. R. \& J. E. WRIGHT. 1975. Clave para el reconocimiento en cultivo de las especies xilófagas de Basidiomycetes argentinae. Rev. Invest. Agrop. INTA, S V, Patología Vegetal 12: 77-87.

DESCHAMPS, J. R. \& J. E. WRIGHT. 1997. Patología forestal del Cono Sur de América. Editora Orientación Gráfica, Buenos Aires.

FARR, D. F., G. F BILLS,G. P.CHAMURIS \& A. Y. ROSSMAN. 1989. Fungi on plants and plant products in the United States. APS Press, Saint Paul.

GOTTLIEB, A. M., J. E. WRIGHT \& J. MONCALVO. 2002. Inonotus s.1. in Argentina-morphology, cultural characters and molecular analyses. Mycol. Prog. 1: 299-313.

GÖTZ, M., H. NIRENBERG, S. KRAUSE, H. WOLTERS, S. DRAEGER, A. BUCHNER, J. LOTTMANN, G. BERG \& K. SMALLA. 2006. Fungal endophytes in potato roots studied by traditional isolation and cultivation-independent DNA-based methods. FEMS Microbiol. Ecol.58: 404-413.

GUARro, J., J. GENÉ, C. DE VROEY \& E. GUÉHO.1992. Hormographiella, a new genus of hyphomycetes from clinical sources. Mycotaxon 45: 179-190.

INTINI, M. \& M. L. TELLO. 2003. Comunicación Investigaciones sobre hongos xilófagos de árboles urbanos en Europa: primera cita de Inonotus rickii (Pat.) Reid en España. Bol. San. Veg. Plagas 29: 277-279.

ISAEVA, A. M., S. A. GLUSHAKOVA, A. V. GARBUZ, I. KACHALKIN \& Y. CHERNOV. 2010. Endophytic yeast fungi in plant storage tissues. Biol. Bull. 37: 26-34.

KEIRLE, M. R., D. E. HEMMES \& D. E. DESJARDIN. 2004. Agaricales of the Hawaiian Islands. 8. Agaricaceae: Coprinus and Podaxis; Psathyrellaceae: Coprinopsis, Coprinellus and Parasola. Fungal Divers. 15: 33-124.

KÜES, U. 2000. Life history and developmental processes in the Basidiomycete Coprinus cinereus. Microbiol. Molec. Biol. Rev. 64: 316-353.
LEVIN, L. L. PAPINUTTI \& F. FORCHIASSIN. 2004. Evaluation of Argentinean white rot fungi for their ability to produce lignin-modifying enzymes and decolorize industrial dyes. Bioresource Technol. 94: 169-176.

MARTIN, R., R. O. GAZIS, D. SKALTSAS, P. CHAVERRI \& D. S. HIBBETT. 2015. Unexpected diversity of basidiomycetous endophytes in sapwood and leaves of Hevea. Mycologia 107: 284-297.doi: 10.3852/14-206.

MARULANDA, A., J. M. BAREA \& R. AZCÓN. 2009. Stimulation of plant growth and drought tolerance by native microorganisms (AM fungi and bacteria) from dry environments: mechanisms related to bacterial effectiveness. J. Plant Growth Regul. 28: 115-124.

MELO, I., P. RAMOS \& M. F. F. CAETANO. 2002. First record of Inonotus rickii (Basidiomycetes, Hymenochaetaceae) in Portugal. Port. Acta Biol. 20: 265-269.

NAKASONE, K. K. 1990. Cultural studies and identification of wood-inhabiting Corticiaceae and selected Hymenomycetes from North America. Mycologia Memoir 15: 1-412.

NOBLES, M. K. 1965. Identification of cultures of wood-inhabiting Hymenomycetes. Can. J. Bot. 43: 1097-1139.

NOBLES, M. K. \& V. J. NORDIN.1955. Studies in woodinhabiting hymenomycetes. II. Corticium vellereum Ellis and Cragin. Can. J. Bot. 33: 105-112.

OSES, R., S. VALENZUELA, J. FREER, E. SANFUENTES \& J. RODRIGUEZ. 2008. Fungal endophytes in xylem of healthy Chilean trees and their possible role in early wood decay. Fungal Divers. 33: 76-87.

PAJOT, H. F., L. I. C. FIGUEROA, J. F. T. SPENCER \& J. I. FARIÑA. 2008. Phenotypical and genetic characterization of Trichosporon sp. HP-2023. A yeast isolate from Las Yungas rainforest (Tucumán, Argentina) with dye-decolorizing ability. Antonie van Leeuwenhoek 94: 233-244.

PARFITT, D., J. HUNT, D. DOCKRELL, H. J. ROGERS \& L. BODDY. 2010. Do all trees carry the seeds of their own destruction? PCR reveals numerous wood decay fungi latently present in sapwood of a wide range of angiosperm trees. Fungal Ecol. 3: 338-346.

PINRUAN, U., N. RUNGJINDAMAI, R. CHOEYKLIN, S. LUMYONG, K. D. HYDE \& E. G. JONES. 2010. Occurrence and diversity of basidiomycetous endophytes from the oil palm, Elaeis guineensis in Thailand. Fungal Divers. 41: 71-88.doi http:// dx.doi.org/10.1007/s13225-010-0029-1

PROMPUTTHA, I., S. LUMYONG, V. DHANASEKARAN, E. H. C. MCKENZIE,K. D. HYDE \& R. JEEWON. 2007. A phylogenetic evaluation of whether endophytes become 
saprotrophs at host senescence. Microb. Ecol. 53: 579-590.

RAMOS BONONI, V. L., A. KLEBER MORBECK DE OLIVEIRA, J. RATIER DE QUEVEDO \& A. DE MELlO GUGliotTA. 2008. Fungos macroscópicos do Pantanal do Rio Negro, Mato Grosso do Sul, Brasil. Hoehnea 35: 489-511.

ROBLES, C. A., C. C. CARMARÁN \& S. E. LOPEZ. 2011. Screening of xylophagous fungi associated with Platanus acerifolia in urban landscapes: Biodiversity and potential biodeterioration. Landscape Urban Plan. 100: 129-135.

ROBLES, C. A., S. E. LOPEZ, P. D. MC CARGO \& C. C. CARMARÁN. 2015. Relationships between fungal endophytes and wood-rot fungi in wood of Platanus acerifolia in urban environments. Can. J. For. Res. 45: 929-936.doi: 10.1139/cjfr-2014-0560.

SCHULZ, B., C. BOYLE. 2006. What are endophytes? En: SCHULZ, B., C. BOYLE \& T. N. SIEBER (eds), Microbial Root Endophytes, pp. 1-13. SpringerVerlag, Berlín.

SCHWARTZ, M. W., J. D. HOEKSEMA, C. A. GEHRING, N. C. JOHNSON, J. N. KLIRONOMOS, L. K. ABBOTT \& A. PRINGLE. 2006. The promise and the potential consequences of the global transport of mycorrhizal fungal inoculum. Ecol. Lett. 9: 501-515.

SEIFERT, K. A., G. MORGAN-JONES, W. GAMS \& B. KENDRICK. 2011. The genera of Hyphomycetes. CBS-KNAW Fungal Biodiversity Centre, Utrecht.

STALPERS, J. A. 1978. Identification of wood-inhabiting fungi in pure culture. Stud. Mycol. 16: 1-248.

VAN OORSCHOT, C. A. N. 1980. A revision of Chrysosporium and allied genera. Stud. Mycol. 20: $1-89$.

WRIGHT, J. E. \& C. L. IACONIS. 1955. Estudios sobre Basidiomycetes III. "Polyporus rickil" f. sp. "negundis" sobre arces vivos. Rev. Invest Agri. 9: 97-109.

Recibido el 17 de junio de 2015, aceptado el 14 de octubre de 2015. 
